\title{
Quantitative thermal imperfection definition using non-destructive infrared thermography on an advanced DEMO divertor concept
}

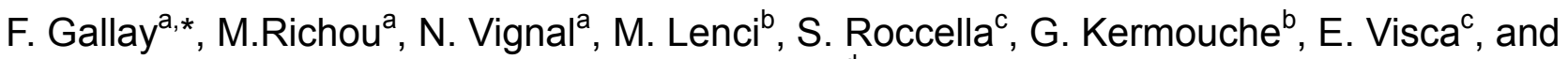 \\ J.H. You ${ }^{d}$, \\ ${ }^{a}$ CEA, IRFM, F-13108 Saint-Paul-Lez-Durance, France \\ ${ }^{b}$ École nationale supérieure des mines de Saint-Étienne, Centre SMS CNRS UMR 5307 Laboratoire \\ Georges FRIEDEL, 158 cours Fauriel, CS 62362 \\ ${ }^{c}$ ENEA, Unità Tecnica Fusione, ENEA C. R. Frascati, via E. Fermi 45, 00044 Frascati, Italy \\ ${ }^{d}$ Max Planck Institute for Plasma Physics, Boltzmann Str. 2, 85748 Garching, Germany
}

The future DEMO divertor is currently under conceptual design within the European Consortium. In this regard, several concepts have been proposed and mock-ups have been fabricated to investigate their thermo-mechanical behaviour. Indeed, as a key plasma facing component, the divertor will have to withstand extreme thermal loads (up to $20 \mathrm{MW} / \mathrm{m}^{2}$ during slow transient events) and will have to be able to exhaust a large amount of heat. The presence of structural defects in the component may significantly affect the thermal response and must therefore be considered. Nondestructive technique based on infrared thermography is proposed here to detect defects in mock-ups where graded material was used as an interlayer between the heatsink material and the armor material. Two methods to characterize the size, the location of such defects are presented. It was shown that finite element analysis combined with experimental data from infrared thermography, provides accurate means to assess quantitatively the size and position of thermal imperfections.

Keywords: DEMO, Divertor, Infrared thermography, non-destructive examination, Plasma-facing component

\section{Introduction}

In the framework of the EUROfusion Consortium, a program of conceptual design activities for the European DEMO reactor [1] was launched in 2014. One of the plasma-facing components (PFCs) concerned by these activities is the DEMO divertor target [2] which will have to withstand high thermal loads (up to $20 \mathrm{MW} / \mathrm{m}^{2}$ during slow transient events). In order to match the relevant structural design criteria in such a harsh loading environment, new design concepts are currently under development and are being qualified with the manufacturing of various mock-ups [3]. In order to ensure the integrity of these mock-ups, their thermal and mechanical behaviours should be assessed in advance. In particular, structural imperfections such as cracks or a low bonding quality between the armor material and the heat sink material may significantly lower the performance of the divertor under heat loads.

Non-destructive testing [4] based on infrared (IR) thermography has proven to be a relevant technique to detect such imperfections [5-8]. In this regard, the CEA has developed its own IR thermography test bed SATIR (named after the French acronym for the IR acquisition and data processing device) to measure the heat exhaust capability of plasma facing components. Within the work package 'WP-DIV', and for one of the developed concept [9], 94\% of the tested structures with using SATIR test bed have shown a relatively good thermal response to external thermal solicitation. However, a delay in thermal response was sometimes observed, indicating the presence of structural defects in the component.
The current work aims at studying these defects in terms of size and position, and at understanding how they can affect the ability of PFCs to exhaust heat, a key property for the qualification program [5]. For this purpose, a first method based on IR data treatment was developed [6]. However, this method requires (for each tested concept) a calibration with the introduction of manufactured artificial defects in the component, for which sizes and position are known [5-8]. Because of the numerous concepts proposed in WP-DIV, this method may be time and money consuming.

Therefore, another method based on the correlation of numerical simulation and SATIR results was investigated here. These two methods were applied to a specific design concept developed at the CEA [9] which uses graded material as an interlayer. Metallographic examinations and ultrasonic tests [10] were also carried out to characterise structural imperfections.

\section{Description of the mock-ups}

For DEMO divertor, the armor material that has been envisaged is tungsten. Copper alloy $(\mathrm{CuCrZr})$ has been chosen as baseline structural material for the cooling pipe because of its high thermal conductivity and mechanical properties at foreseen operation temperature $\left(150{ }^{\circ} \mathrm{C}\right.$ to $\left.350^{\circ} \mathrm{C}\right)[11]$.

Various kinds of new interlayer materials are considered to replace pure copper (CuOFHC) used in ITER divertor concept as a compliant layer between tungsten (W) and $\mathrm{CuCrZr}$ [3]. One of the developed concepts, which is studied here, uses Functionally Graded Material (FGM) as an interlayer [9]. For this concept, the $\mathrm{CuCrZr}$ cooling tube was joined to $\mathrm{W}$ using a very thin graded 
$\mathrm{W} / \mathrm{Cu}$ film as bonding agent. One mock-up consists of 10 tungsten blocks (size $4 \mathrm{~mm} \times 22 \mathrm{~mm} \times 23 \mathrm{~mm}$ ) with an adhesive deposit of FGM in the inner part (thickness $\sim 25 \mu \mathrm{m}$ ) and assembled with a $\mathrm{CuCrZr}$ tube. The geometry of one tungsten monoblock is shown in Fig.1.a.

Seven mock-ups of this concept have been manufactured (mock-up M0 to M6) and tested using SATIR, Ultrasonic tests (UT), and high heat flux tests [9]. Monoblocks are numbered from 1 to $10, \mathrm{M} 0-1$ to $\mathrm{M} 0-10$ for mock-up 0 for example.



(b)

Fig. 1: Schematic view of a monoblock with graded material as interlayer (for DEMO divertor concept) (a) and mesh used for the finite element modeling of this monoblock where a standard defect is also located at the $\mathrm{W} / \mathrm{CuCrZr}$ interface (b)

\section{Defect detection and characterization methods}

\subsection{SATIR}

The SATIR facility is an active Infrared thermography test bed based on the heat transient method [7]. Developed at the CEA, this installation is able to determine the heat exhaust capability of components and to detect potential thermal imperfections that may be present in PFCs. This technique is based on thermal inspection of the component during a fast temperature variation (105 to $10{ }^{\circ} \mathrm{C}$ in few $\mathrm{ms}$ ) of water flowing in the cooling channel of a target mock-up. The surface temperature is measured during the transient phase via an IR camera (CEDIP JADE II $\lambda=3-5 \mu \mathrm{m}$ ). In this test, the transient evolution of the surface temperature of the tested mock-up is compared with that of a reference mock-up being defect-free. The maximum temperature difference for each pixel (DtRef) occurring during the cooling transient phase is calculated. For each examined face of the component, corresponding DtRef maps are extracted and the maximum of the DtRef values is referenced as DtRef $\max _{\text {max }}$ A slow response of the surface temperature accounts for a high thermal resistance, likely to come from a bad bonding quality between the different layers of materials or the presence of defects in the materials themselves.

\subsection{Method 1: EQuivalent thermal Imperfection (EQI)}

To assess the size and position of the thermal imperfections, a first method relies on the implementation of a threshold (called here later EQI threshold) on DtRef values [6]. DtRef values higher than this EQI threshold may be attributed to the presence of a thermal imperfection located on the thermal path from the cooling tube to the observed external surface. These thermal imperfections are most likely due to interlayer/CuCrZr interface debonding [5]. Consequently, DtRef maps are attributed to an EQI localized at the surface of the external $\mathrm{CuCrZr}$ tube and described with an extension $(\Delta \theta)$ and position $(\theta)$ of a probable thermal imperfection. To define the EQI extension and position, 2D DtRef maps of all faces are geometrically projected onto the external $\mathrm{CuCrZr}$ tube map and the EQI threshold is applied [6]. The defect extension is obtained from this map and corresponds to the angle interval between the two positions which are related to DtRef equals to EQI threshold. Defect position is localized at the center of this deduced extension.

One limit of the EQI method is that, for each of the tested geometries, the EQI threshold must be assessed preliminary with dedicated calibration and examination of artificially manufactured calibrated defects [5] [6] [7] [8][5-7]. With the several geometries developed in the WP-DIV project, and thereby the many artificial defects to be manufactured, this calibration appears complex to achieve. For this reason, and based on a past experience [7], the EQI threshold is here set to the value of $8^{\circ} \mathrm{C}$ without performing any calibration.

\subsection{Method 2: FEM modeled Imperfection (FEMI)}

Another approach, the FEMI method, consists in comparing finite element method (FEM) modeling with SATIR experimental results. The most important benefit compared to the EQI method is that the FEMI method is not linked to an EQI threshold.

Nevertheless, as a preliminary study, and for comparison purpose with EQI method, the thermal imperfection position $(\theta)$ and extension $(\Delta \theta)$ are only assessed for monoblocks with DtRef values higher than EQI threshold (i.e: $8^{\circ} \mathrm{C}$ ).

For SATIR experimental data extraction, 2D DtRef maps of all faces are first geometrically projected onto the external $\mathrm{CuCrZr}$ tube map. Then, for the purpose of the analysis, the experimental 1D DtRef profile is extracted at the monoblock depth including DtRef $\mathrm{max}_{\text {max }}$.

For modeled data extraction, SATIR experiments of a defective monoblock and a defect-free monoblock are simulated using 2D FEM modeling with ANSYS V17.2. Implemented geometries with and without defect are presented in Fig. 1. The thin FGM thickness of $\sim 25 \mu \mathrm{m}$ has been neglected due to its negligible effect on the surface temperature behavior. For defective monoblock geometries, the standard defects implemented have a thickness of $300 \mu \mathrm{m}$ (see Fig. 1) with air thermal properties. The choice of modeling standard defects (thickness and localization) has been made since 
imperfections are most likely of this thickness and frequently positioned at this interface [5]. In the following, such defects will be called standard defects. All the components are assumed to be constituted with the same material characteristics. Convection coefficient is supposed to be applied as an abrupt step from $110^{\circ} \mathrm{C}$ to $10^{\circ} \mathrm{C}$ which may not be representative of the water temperature real evolution during the SATIR test. Radiation and convection on external surfaces have been neglected. The surface temperature for each node is obtained for geometries with and without thermal imperfection. The maximum temperature difference between those two geometries and for each node occurring during the cooling transient phase of the $\mathrm{W}$ surface is calculated and projected at the $\mathrm{CuCrZr}$ /interlayer interface allowing us to define modeled 1D DtRef profiles.

Since differences exist between experimental and numerical DtRef ${ }_{\max }$ data, experimental and modeled 1D DtRef profiles are normalized. These differences may be due to water parameters implemented in simulation which are different from the experimental ones. To improve accuracy of the model a prospect could be to integrate in the model the water temperature evolution related to the studied experimental case. Finally, the FEMI position $(\theta)$ is assessed at the angle corresponding to the peak value of experimental 1D DtRef profiles. The FEMI extension $(\Delta \theta)$ corresponds to the modeled defect extension $\Delta \theta$ for which modelled 1D DtRef profile show most-fitted curve with the experimental 1D DtRef profile. Between each modeled defect, an extension step of $10^{\circ}$ is used (see Fig. 2). In order to fit profiles, least squares adjustments are performed between 1D DtRef profiles and experimental 1D DtRef profile. For experimental 1D DtRef profile some mismatches of DtRef values are observed at the junctions between 2 faces. Indeed, for each face a SATIR test is performed. Testing parameters may be different for these two testing. This may induce a DtRef inhomogeneity at the angle related to the junction between two faces. Consequently, for the adjustments, a restricted range of angular values is arbitrary used. Only DtRef values related to angular positions of one face are considered. This range of angular values corresponds to the projected angles of the one particular face containing DtRef $f_{\max }$.

\section{Experimental results}

To evaluate EQI \& FEMI methods, results for two monoblocks of mock-up M0 are firstly presented and correlated with metallographic examinations. Afterwards, these methods are applied to other mock-ups (M1 to M6) and correlated with ultrasonic tests results as for these mock-ups no metallographic examinations were performed.

\subsection{EQuivalent thermal Imperfection (EQI) results}

With the EQI method applied to mock-up M0, two monoblocks are evaluated as presenting some thermal imperfections: M0-1, with a thermal imperfection of extension $\Delta \theta=80^{\circ}$ and positioned at $\theta=35^{\circ}$ and $\mathrm{M} 0-9$, with a thermal imperfection of extension $\Delta \theta=20^{\circ}$ and positioned at $\theta=-105^{\circ}$. Corresponding results are summarized in Table 1.

\subsection{FEM modeled Imperfection (FEMI) results}

For both monoblocks, modeled 1D DtRef profiles show curves centered on $\theta$ (see Fig. 2). For M0-1 and M0-9, experimental 1D DtRef profiles and corresponding peak value positions show (see Fig. 2) imperfections positioned at respectively $\theta=50^{\circ}$ and $\theta=-110^{\circ}$.

Adjustments between experimental and modeled profiles allow to assess extensions of $\Delta \theta=260^{\circ}$ for M0-1 and of $\Delta \theta=85^{\circ}$ for M0-9 (Table 1). For M0-1 and for the chosen adjustment range of angles $\left[\theta=45^{\circ} ; 135^{\circ}\right]$, a good agreement between modeled and experimental profile is observed (sum of squared deviation $=0,059$ ). However, for the range $\left[\theta=-135^{\circ} ; 45^{\circ}\right]$, important differences between modeled and experimental profiles are observed (up to $13 \%$ ). The difficulty to fit profiles might be explained by differences between modeled and real defects in terms of shape, thickness or radial position. For this reason, when experimental and modeled profiles don't fit, and in addition to testing parameters that may be different for these two tested faces, a non-standard defect can be foreseen. For M0-9, and for the chosen adjustment range of angles $\left[-135^{\circ}\right.$; $45^{\circ}$ ], a good correspondence between modeled and experimental profiles is observed (sum of squared deviation $=0,058)$.



Fig. 2: Normalized DtRef profiles obtained with finite element method (FEM) and with experimental data for monoblocks M0-1 (top) and M0-9 (bottom)

\subsection{Definition of the thermal imperfection reference size}

Definition of the thermal imperfection reference size is performed using different techniques (Visual observation after metallographic examination for M0 and ultrasonic testing for M1 to M6).

For mock-up M0, monoblocks M0-1 and M0-9 are examined with an optical microscope (Olympus BX60M) and a binocular magnifier (Olympus SZX9). With these metallographic observations two kinds of damage are highlighted. For M0-1, a thin crack propagating circumferentially, at a radial distance of $1.5 \mathrm{~mm}$ from the $\mathrm{CuCrZr} / \mathrm{W}$ interface with an extension of $\Delta \theta=300^{\circ}$ and positioned at $\theta=70^{\circ}$ is detected. For M0- 
9 , cavities (size $\sim 500 \mu \mathrm{m}$ ) and cracks are emphasized, at a distance $<200 \mu \mathrm{m}$ with a total extension of $\Delta \theta=105^{\circ}$. Defect is positioned at $\theta=-117^{\circ}$ (see Fig. 3).

Thermal imperfection reference sizes and positions are summarized in Table 1.
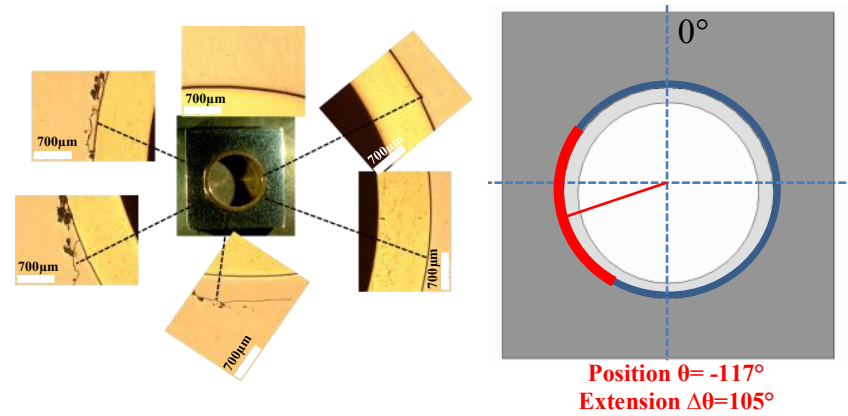

Fig. 3: Optical micrographs of the $\mathrm{W} / \mathrm{CuCrZr}$ joint in M0-9 monoblock (a) and representation of the observed defect (b)

\subsection{Evaluation of EQI and FEMI methods}

In this part, positions and extensions of thermal imperfections are assessed for all tested mock-ups (see Table 1). Results are compared with the thermal imperfection reference sizes.

For M0-1, the FEMI method data processing shows that modeled data are difficult to fit with experimental data. Consequently, a non-standard defect is foreseen and finally confirmed by metallographic examination. Despite this, the size assessment accuracy is improved (from differences of $-70 \%$ with EQI method to $-13 \%$ with FEMI method).

For M0-9, comparisons of metallographic observations with EQI results (see Table 1) show significant underestimation of the thermal imperfection extension $(-81 \%)$. This difference shows that applying the EQI method with an arbitrary threshold may generate important errors on the thermal imperfection size definitions.

For M2-1, both methods show consistent results with UT examination with an extension of $\Delta \theta=360^{\circ}$.

For M2-10, FEMI method result shows an improvement of extension assessment accuracy compared to EQI method.

Finally, for M6-10 FEMI method result retains an important difference of $22 \%$ with regard to the reference. For this monoblock, data processing also shows important differences between modeled and experimental data which may be explained by the fact that a defect is localised in tungsten [9] but modeled at interface.

To conclude, for the tested components, the FEMI method allows an accurate assessment (with maximal differences of $\sim 19 \%$ compared to the reference) of the thermal imperfection extension when thermal imperfection is positioned at the interface.

Despite the numerous attempts to model adequate defect size, it was observed in this study that, if experimental and modeled profiles don't fit, defects may not be modeled representatively as the real defect. Consequently, with the FEMI method, the presence of non-standard defects can be foreseen and eventually confirmed with achieving further tests such as ultrasonic testing in the whole monoblock thickness (and not only at interface).

One prospect is to apply the FEMI method to all monoblocks presenting Gaussian DtRef experimental profiles typical of a monoblock with thermal imperfection (see Fig. 2).

\begin{tabular}{|c|c|c|c|c|c|}
\hline Monoblock & $\begin{array}{c}\text { EQI } \\
(\Delta \theta, \theta)\end{array}$ & $\begin{array}{c}\% \\
\text { (EQI vs } \\
\text { Ref) }\end{array}$ & $\begin{array}{l}\text { FEMI } \\
(\Delta \theta, \theta)\end{array}$ & $\begin{array}{c}\% \\
\text { (FEMI } \\
\text { vs Ref) }\end{array}$ & $\begin{array}{l}\text { Ref. } \\
(\Delta \theta, \theta)\end{array}$ \\
\hline M0-1 & $80^{\circ}, 35^{\circ}$ & $-70 \%$ & $260^{\circ}, 50^{\circ}$ & $-13 \%$ & $300^{\circ}, 70^{*}$ \\
\hline M0-9 & $20^{\circ},-105^{\circ}$ & $-81 \%$ & $85^{\circ},-110^{\circ}$ & $-19 \%$ & $105^{\circ},-117^{\circ} * *$ \\
\hline M2-1 & $360^{\circ}$ & $0 \%$ & $360^{\circ}$ & $0 \%$ & $360^{\circ} * *$ \\
\hline M2-10 & $300^{\circ}, 140^{\circ}$ & $10 \%$ & $280^{\circ}, 140^{\circ}$ & $3 \%$ & $270^{\circ}, 145^{\circ * *}$ \\
\hline M6-10 & $135^{\circ}, 70^{\circ}$ & $-22 \%$ & $135,70^{\circ}$ & $-22 \%$ & $173^{\circ}, 65^{\circ *}$ \\
\hline \multicolumn{6}{|c|}{$\begin{array}{l}* \text { defect observed in the bulk tungsten } \\
* * \text { defect located at the interface }\end{array}$} \\
\hline
\end{tabular}

Table 1: Extension and position $(\Delta \theta, \theta)$ of thermal imperfections obtained with reference testing, EQI and FEMI methods and comparisons of $\Delta \theta$ obtained with reference testing and EQI method (\% (EQI vs Ref.)) and obtained with reference testing and FEMI method (\% (FEMI vs Ref.))

\section{Conclusion}

In summary, infrared thermography using SATIR test bed was shown to be a well-suited technique for evaluating the heat exhaust capability of plasma facing components which may be weakened by the presence of thermal imperfections at material interfaces. The quantification of the position, size and origin of thermal imperfections with the use of non-destructive techniques is an important topic to be addressed. In this work, the reliability of SATIR tests combined with one existing (EQI) method in order to assess quantitatively size and position of thermal imperfections has been evaluated. It was observed that this method presents some significant inaccuracies for the extension determination (differences up to $-81 \%$ compared to the reference). In order to reduce these differences and to avoid costly calibration, the development of a new method (FEMI), based on correlations between numerical modeling and SATIR experimental results, was carried out. In some instances, it was shown that the accuracy of the extension assessment can be improved. Also, this FEMI method does not require any manufacturing process or threshold determination and could potentially be applied for any kind of geometry and known material.

To conclude, FEMI method appears to be relevant to detect and characterize thermal imperfections for the analyzed monoblocks and when thermal imperfections are located at the interface. When important differences between experimental and simulated DtRef data are observed, the thermal imperfection may be different from the one modeled in terms of shape, thickness or location in the monoblock. In this case an additional non-destructive examination such as ultrasonic tests and data merging [12] can also be used. 


\section{Acknowledgments}

This work has been carried out within the framework of the EUROfusion Consortium and has received funding from the Euratom research and training programme 2014-2018 under grant agreement No 633053. The views and opinions expressed herein do not necessarily reflect those of the European Commission

\section{References}

[1] Federici G et al 2016 Fusion Eng. Des. 1091464

[2] You J H et al 2016 Nuclear materials and Energy 9171

[3] You J H et al 2016 Fusion Eng. Des. 1091598

[4] Merola M et al 2002 Fusion Eng. Des. 61141

[5] Escourbiac F, Richou M, Guigon R, Constans S, Durocher A, Merola M, Schlosser J, Riccardi B and Grosman A 2009 Physica Scripta 2009 T138

[6] Durocher A, Escourbiac F, Richou M, Vignal N, Merola M, Riccardi B, Cantone V and Constans S 2009 Fusion Eng. Des. 84314

[7] Richou M, Escourbiac F, Missirlian M, Vignal N, Cantone V and Riccardi B 2011 J. Nucl. Mater. 417581

[8] Vignal N, Desgranges C, Cantone V, Richou M, Courtois X, Missirlian M and Magaud P 2013 Fusion Eng. Des. 881818

[9] Richou M, Gallay F, Chu I, Li M, Magaud P, Missirlian M, Rocella S, Visca E and You J H 2017 Fusion Eng. Des. http://dx.doi.org/10.1016/j.fusengdes.2017.03.087

[10] Roccella S, Cacciotti E, Escourbiac F, Pizzuto A, Riccardi B, Tatì A, Varone P and Visca E 2009 Fusion Eng. Des. 841639

[11] Li Puma A, Richou M, Magaud P, Missirlian M, Visca E and Ridolfini V P 2013 Fusion Eng. Des. 881836

[12] Richou M, Durocher A, Medrano M, Martinez-Oña R, Moysan J and Riccardi B 2009 Fusion Eng. Des. 841593 